\title{
Review: evidence is unclear that preventive home visits for elderly people in the community improve mortality or health outcomes
}

van Haastregt JC, Diederiks JP, van Rossum E, et al. Effects of preventive home visits to elderly people living in the community: systematic review. BMJ 2000 Mar 18;320:754-8.

QUESTION: For elderly people living in the community, are preventive home visits effective for improving physical and psychological function and for reducing falls, admission to institutions, and mortality?

\section{Data sources}

Studies were identified by searching Medline (1966 to May 1999), EMBASE/Excerpta Medica (1989 to March 1999), and the Cochrane Controlled Trials Register with the terms geriatric assessment, home visit, health visit, and health screening combined with the terms aged, prevent, screen, health education, or health promotion with various term endings. Bibliographies of studies and review articles were scanned.

\section{Study selection}

Randomised controlled trials were selected if elderly people who were $\geq 65$ years of age and living in the community were evaluated; home visit interventions were tested; interventions were designed to prevent or reduce problems and risks related to aging; problems or resources in $\geq 2$ medical, functional, psychosocial, or environmental categories were assessed and specific recommendations were made for reducing, treating, or preventing observed or potential problems; visits were not exclusively concerned with a specific illness or a hospital discharge; and data were available on $\geq 1$ outcome (physical or psychosocial function, falls, admissions to institutions, or mortality).

\section{Data extraction}

Data were extracted on study quality, participant characteristics, interventions, follow up, and outcomes.

\section{Main results}

244 abstracts were screened, 29 articles were evaluated, and 15 studies met the inclusion criteria. Mean quality score was $54 \%$ of the maximum score (range $29 \%$ to $68 \%$ ). Substantial variation was seen in the interventions. 6 studies included only participants who were $\geq 75$ years of age, 9 interventions lasted $>2$ years, and 7 studies used $\geq 2$ visits/year. 94 outcome measures were evaluated: 8 trials reported $\geq 1$ improved outcome, 5 reported no improved outcomes, and no trials reported negative results. 12 studies investigated physical function: 5 reported that $\geq 1$ measure had improved ( 2 in basic or instrumental activities of daily living; 2 in self rated health or health problem status; and 1 in balance, gait, and toilet transfer skills). 8 studies investigated psychosocial function: 1 showed an improvement in attitude to own aging, loneliness, isolation, and emotional reaction. 6 studies investigated the number of falls: 2 showed reductions. 7 studies investigated admissions to institutions: 2 showed reductions -1 in hospital admissions and 1 in permanent admissions to a nursing home. 13 studies evaluated mortality: 3 found lower mortality rates after home visits.

\section{Conclusion}

Although home visits for elderly people were not associated with any negative outcomes, evidence is unclear that such visits improve overall outcomes or specific outcomes of physical or psychosocial function, falls, admissions to institutions, or mortality.

\section{COMMENTARY}

Comprehensive geriatric assessment (CGA) is designed to improve the health of elderly people by evaluating available medical, psychosocial, functional, and environmental resources and by providing targeted interventions to optimise clinical outcomes. Although several forms of CGA have evolved, this review by van Haastregt et al focuses on community based assessment using home visits.

A previous meta-analysis by Stuck $e t$ al found that home assessment services decreased long term mortality by $14 \%$, increased the number of people remaining in their homes, and reduced hospital admissions. ${ }^{1}$ However, the review included only 5 studies with substantial methodological variation, calling into question the strength of the conclusions. The current study builds on this knowledge by examining randomised controlled trials of home assessment services completed since the publication of the first analysis.

It is disappointing but not surprising that van Haastregt $e t$ al found that home visits had limited influence on clinical outcomes. The studies differed in several important aspects, including the targeted population, nature of the home evaluation and subsequent interventions, compliance with recommendations, and duration of follow up. Thus, excluding home visits from geriatric assessment on the basis of available evidence is inappropriate.

The lack of consistent benefit of CGA and the cost and resource use involved in providing these services highlight the critical need for careful examination to determine which components of home based geriatric assessment and intervention are effective. Future efforts should also focus on identifying high risk groups likely to benefit from home assessment services and assessing interventions that could affect clinically important outcomes.

Philip A Masters, MD, MSW Pennsylvania State University Hershey, Pennsylvania, USA

1 Stuck AE, Siu AL, Wieland GD, Adams J, et al. Comprehensive geriatric assessment: a meta-analysis of controlled trials. Lancet 1993;342:1032-6. 